\title{
A Planar Glass/Si Micromachining Process for the Heat Exchanger in a J-T Cryosurgical Probe
}

\author{
$\underline{\text { Weibin Zhu }}^{1}$, Daniel W. Hoch ${ }^{2}$, Gregory F. Nellis ${ }^{2}$, Sanford A. Klein ${ }^{2}$, Yogesh B. Gianchandani ${ }^{1}$ \\ ${ }^{I}$ Department of Electrical Engineering and Computer Science, University of Michigan, Ann Arbor \\ ${ }^{2}$ Department of Mechanical Engineering, University of Wisconsin, Madison
}

\begin{abstract}
This paper reports a lithography-based microfabrication process and the preliminary experiments for a recuperative heat exchanger intended for use in a Joule-Thomson (J-T) cryosurgical probe. The heat exchanger must maintain high stream-to-stream thermal conductance while restricting parasitic stream-wise (axial) conduction losses. Rows of fins composed of high conductivity silicon are bonded onto a $100 \mu \mathrm{m}$ thick base plate composed of low conductivity Pyrex glass. The heat exchanger has a footprint of $6 \times 1.5 \mathrm{~cm}^{2}$ and $2.5 \mathrm{~mm}$ thickness, and is fabricated using a 5 mask process. The fabrication process involves anodic bonding, deep reactive ion dry etching, glass frit bonding, electrochemical discharge drilling and several other steps, along with special features that compensate for manufacturing tolerances. Preliminary experiments include measurement of the thermal effectiveness in an ice-water bath, as well as evaluation of self-cooling with butane. The data suggest an optimal area of $0.2 \mathrm{~mm}^{2}$ for the expansion orifice. The primary performance constraint is imposed by the compromise between mechanical robustness and transverse conductance of the thin glass plate that separate the high pressure and low pressure sides.
\end{abstract}

\section{INTRODUCTION}

Cryosurgery has been used to treat several types of cancers including prostate cancer and liver cancer in the past three decades. In this medical procedure, cancerous tumors are locally destroyed by exposing pathological tissues to repeated freeze/thaw cycles [1]. The ablated tissue is subsequently absorbed or sloughed by the body. By avoiding excision, operative blood loss and discomfort are minimized. Even in the cases where excision is required, the growth of tumor cells that might escape during resection can be prevented by a pre-treatment on the tumors with the cryotherapy.

Cryosurgery, which is competitive with other therapies, has been a standard treatment for cancerous tumors located in easily accessible areas of the body. Recent development of miniature cryoprobes with large refrigeration capacity [2-4] and techniques for real-time monitoring using ultrasound or magnetic resonant imaging [5-7] is allowing cryosurgical techniques to be extended to the treatment of cancers in areas that are not readily accessible [8]. In fact, this effort, which examines the possibility of fabricating cryoprobes with micromachining technologies, is motivated in part by the need of miniaturizing cryoprobes to a size that permits them to be inserted through a small incision. However, the performance of the cyroprobe must not be compromised in this scaling effort because the freeze region that surrounds the cold end of the probe is controlled by the refrigeration power and surface temperature attainable by the tip of the probe. It is known, for example, that temperatures below $-50^{\circ} \mathrm{C}$ are always necrotic for pathological tissue [9-11]. In addition, a rapid cool-down rate [1, 12] between $25-50{ }^{\circ} \mathrm{C} / \mathrm{min}$. and multiple freeze-thaw cycles [13] are preferred to decrease the likelihood of cell survival.

The ultimate goal of this research effort is to develop a fully integrated micromachined cryosurgical probe that has significant advantages over the conventional cryosurgical probe in terms of thermal performance, size, flexibility and cost. The JouleThomson cooling cycle (Fig. 1) can meet these requirements [14]. In this cycle, cold, high-pressure fluid leaving a recuperative heat exchanger expands through a valve, resulting in a temperature drop through the valve if the state of the fluid lies below the inversion curve before expansion. This paper describes the fabrication and preliminary experiments of one possible approach to the micromachined recuperative heat exchanger which utilizes a planar design and 5 wafers in a 5-mask process (Fig. 2).

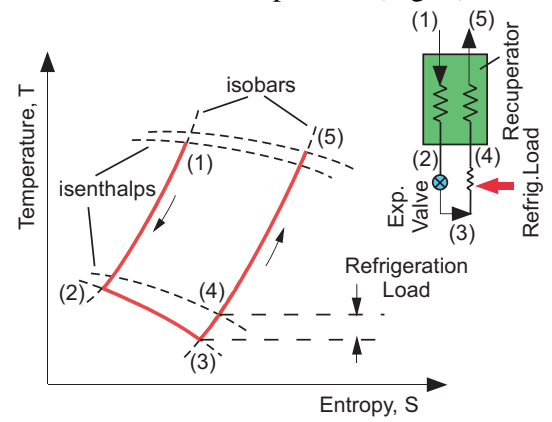

Fig. 1: Joule-Thomson refrigeration cycle. High pressure fluid at state (1) passes through a counter flow recuperative heat exchanger, where it is pre-cooled by the low-pressure fluid returning from the refrigeration load. The cold high-pressure fluid leaving the heat exchanger at state (2) expands through a valve to state (3). The cold, low-pressure fluid is directed through the load heat exchanger where it is warmed by the refrigeration load to state (4) and then is fed back into the heat exchanger.

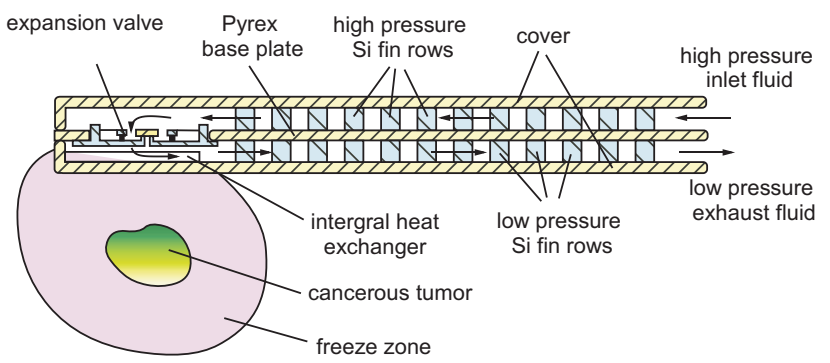

Fig. 2: Schematic diagram of micromachined Joule-Thomson cryosurgical probe. High pressure inlet fluid is obtained from external compressor.

\section{DESIGN AND FABRICATION}

One of the critical challenges in developing a micromachined $\mathrm{J}$-T cooler is that the recuperative heat exchanger must maintain good stream-to-stream heat conductance while restricting parasitic stream-wise conduction losses. This requirement is necessary in order to have a large enthalpy difference between the two streams and thus achieve high cooling performance for the probe. The planar, micromachined recuperative heat exchanger uses rows of fins that are composed of high conductivity silicon that is anodically bonded onto a very thin base plate composed of low 
conductivity Pyrex (Fig. 3). This design is in contrast to conventional cryogenic heat exchangers that use either perforated plate designs with oxygen-free high conductivity (OFHC) copper plates interleaved with stainless steel spacers or finned tube designs that use one or more finned tubes wound on a mandrel. Silicon has thermal conductivity that is similar to OFHC copper, and Pyrex has thermal conductivity that is an order of magnitude less than stainless steel; this combination of very high and low thermal conductivity suggests that a silicon and Pyrex composite heat exchanger will be attractive. In order to allow adequate thermal communication between the streams, preliminary modeling [15] and simulation of an optimized design [16] suggest that for a working pressure of 20 bar, the Pyrex base plate between the high pressure channel and low pressure channel can be no thicker than about $100 \mu \mathrm{m}$ in order to provide adequate refrigeration power.

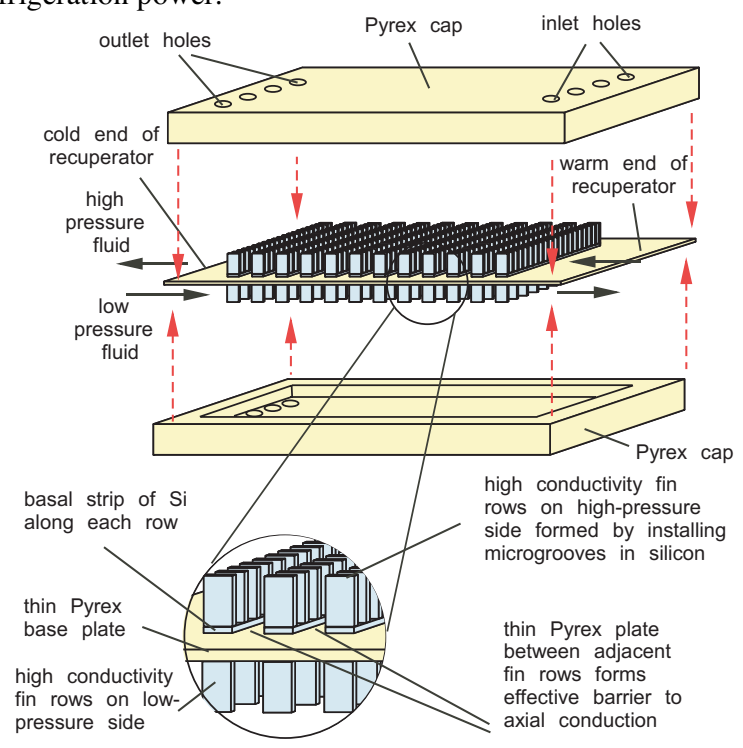

Fig. 3: Fin rows structure of the micro heat exchanger.

There are five masks required for this process (Fig. 4):

1) Two Si wafers of $200 \mu \mathrm{m}$ thickness are bonded to a glass wafer of $100 \mu \mathrm{m}$ thickness. The entire stack is loaded simultaneously into the bonding apparatus and heated to $400^{\circ} \mathrm{C}$, before a $500 \mathrm{~V}$ bonding voltage is applied. By sequentially reversing the polarity of the voltage source, both Si-Pyrex interfaces are bonded one-by-one.

2) A $2 \mu \mathrm{m}$-thick layer of silicon dioxide is deposited on both sides of the $\mathrm{Si}$ surface using plasma enhanced chemical vapor deposition (PECVD). This layer and a subsequent layer of photoresist will serve as masks in a two-step DRIE process.

$3,4)$ The oxide layers on both sides are then patterned by reactive ion etching (RIE).

5) A $10 \mu \mathrm{m}$ thick layer of photoresist is then coated and patterned on the top side. A conformal thick resist layer is used to protect the oxide pattern in the next DRIE step. On the top side of the wafer, Si channels between each fin row are etched down 20 $\mu \mathrm{m}$ by DRIE before the photoresist is stripped. This etch will ultimately lead to the creation of the basal strip along each row that is illustrated in the magnified part of Fig. 3. It is needed in part to compensate for a DRIE artifact that is explained below, but also has the added benefit of strengthening the attachment of the fins to the glass base plate.

6) A $10 \mu \mathrm{m}$ photoresist layer is coated and patterned on the bottom side. On the bottom side of the wafer, Si channels between each fin row are etched down $20 \mu \mathrm{m}$ by DRIE. The photoresist is then stripped.

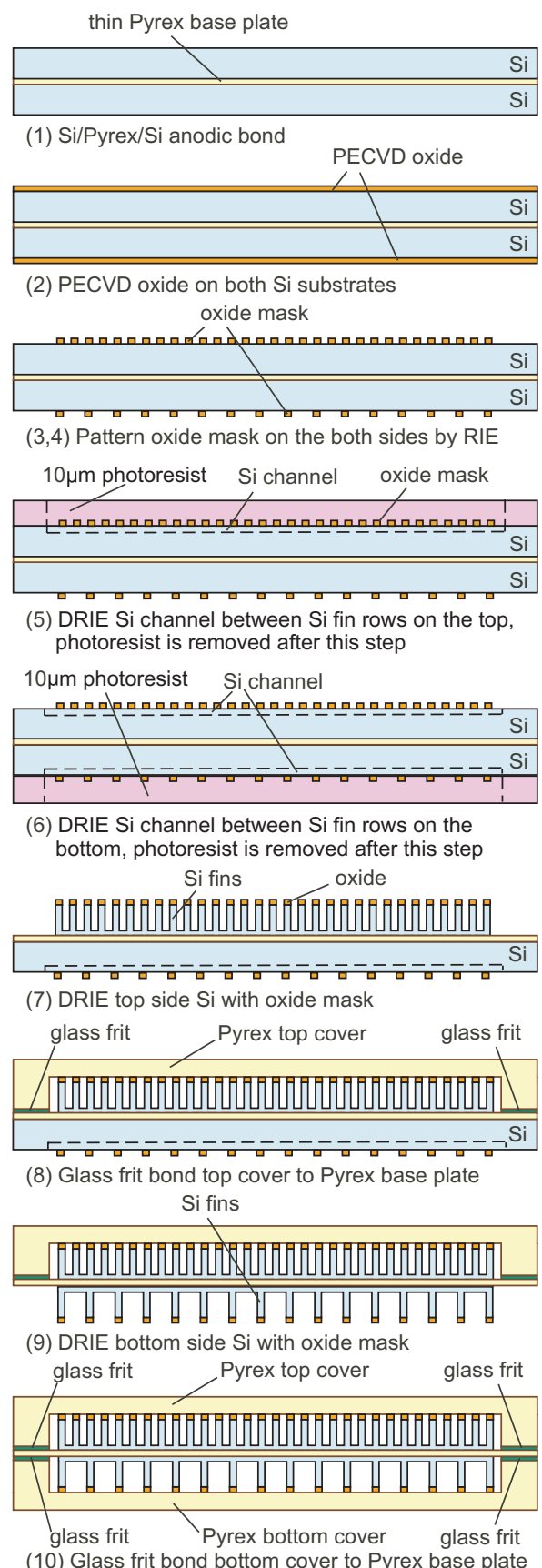

Fig. 4: Fabrication process flow of micro heat exchanger.

7) Fin rows on the top side are etched and defined by DRIE with an oxide mask. This is a high aspect ratio etch of $180 \mu \mathrm{m}$ depth. The etch must clear the narrow regions between rows, etch between the tightly packed fins, and also clear the perimeter of the device, which has no masked features, and etches faster.

Optimizing the DRIE chemistry is one of the significant challenges of the process for three reasons: (a) the relatively large size of the device and the narrow widths of the fins demands high uniformity over a large area; (b) the DRIE step must clear the narrow openings between rows and the wide spaces of the perimeter simultaneously; and (c) the termination of the etch on the glass layer sandwiched between the two Si layers causes a problem known as "footing," which is the lateral spread of the etch profile once the insulating glass is exposed at the bottom of the trench [17]. The two-step DRIE (in steps 5 and 7) is necessary to fabricate separated grooves on the high-pressure side, shown in Fig. 3, and 
compensate for the non-uniform etch rate due to the varying density of the features. Due to micro loading [18, 19], the areas between fins and fin rows with small openings are usually etched more slowly than the area around the device with large openings. Since each fin row must be thermally isolated along the flow direction, the $\mathrm{Si}$ in the gap between fin rows must be completely etched away while the over-etch in the area around the device must be minimized to prevent the footing effect from damaging nearby fins. In step 5, the area between each fin row is etched down 20 $\mu \mathrm{m}$, allowing this densely packed region to be etched through fully during the DRIE in step 7.

8) A glass cap (the fabrication of which is described separately, below) is bonded to the base plate with commercial glass frit tape (G1017, from Vitta Corp.) at $500^{\circ} \mathrm{C}$ so as to construct a sealed chamber on the high pressure side. A significant challenge in this step is patterning of the tape, which cannot be done lithographically. In this effort, because of the large bonding area, the tape was cut and placed on the die manually.

9) A similar DRIE process is completed on the low-pressure side afterward.

10) Another glass cap is bonded onto the bottom side of the base plate using glass frit.

The glass caps are fabricated from $1.1 \mathrm{~mm}$ thick Pyrex wafers. The Pyrex wafer is coated with $500 \AA / 5000 \AA \mathrm{Cr} / \mathrm{Au}$ layer and then patterned with photoresist. Using the metal layer as a mask, the wafer is immersed in the $\mathrm{HF}: \mathrm{HNO}_{3}$ solution to create a recess that is $100 \mu \mathrm{m}$ deep. The photoresist and $\mathrm{Cr} / \mathrm{Au}$ layer are removed after the wet etching. Inlet and outlet holes are drilled with an electrochemical discharge drilling method [20]. This method provides a smooth and debris-free surface, but is a serial method, and is not performed lithographically. An electrode needle is positioned in the proximity of the glass wafer while both are immersed in $\mathrm{NaOH} 30 \%$ wt. solution at room temperature. A bias of $33 \mathrm{~V}$ permits a $1100 \mu \mathrm{m}$ thick glass plate to the perforated in about $10 \mathrm{~s}$. Figure 5 shows a fabricated micro recuperative heat exchanger. The size of this device is $6 \times 1.5 \mathrm{~cm}^{2}$ with $2.5 \mathrm{~mm}$ total thickness.

a)
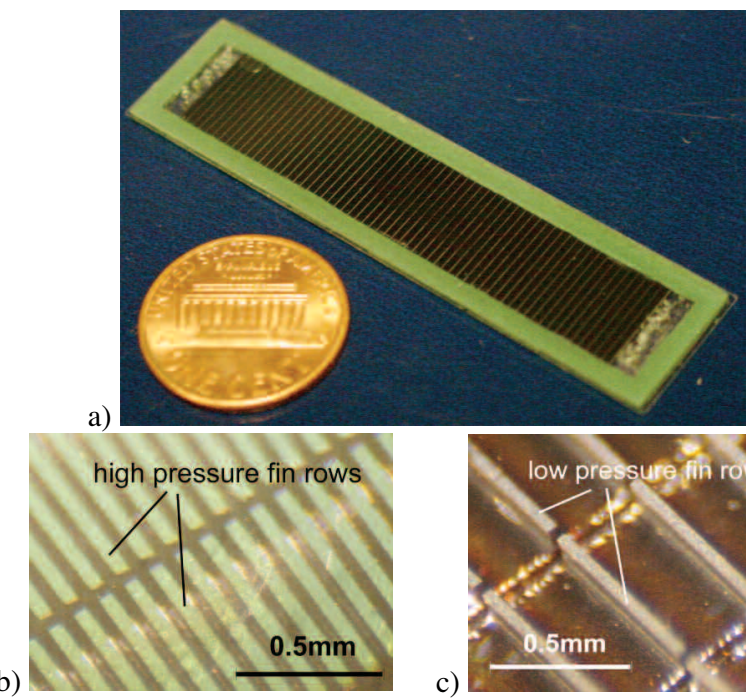

c)

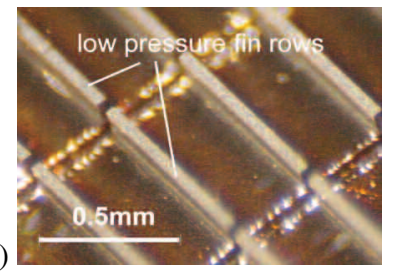

Fig. 5: a) The size of micro recuperator is $6 \times 1.5 \mathrm{~cm}^{2}$ with total $2.5 \mathrm{~mm}$ thickness. Gap between each fin row is $51 \mu \mathrm{m}$. b) High pressure fin rows with $50 \mu \mathrm{m}$ gap, fin size is $50 \times 782 \mu \mathrm{m}^{2}, 200 \mu \mathrm{m}$ high. c) Low pressure fin rows with $345 \mu \mathrm{m}$ gap. Fin size is the same as high pressure fins.

\section{MEASURED RESULTS}

Two types of preliminary tests were performed. The micro heat exchanger was first tested in an ice bath (Fig. 6), with helium gas flowing through one side of the micro heat exchanger and ice water flowing through the other side. At the beginning of the test, the ice water inlet pressure was increased in order to match the helium inlet pressure, so that the pressures on either side of the heat exchanger were approximately balanced. This configuration provided essentially a constant, $0^{\circ} \mathrm{C}$ heat sink on one side so that the thermal effectiveness could be evaluated in this simple limit and compared with the numerical model of the device.

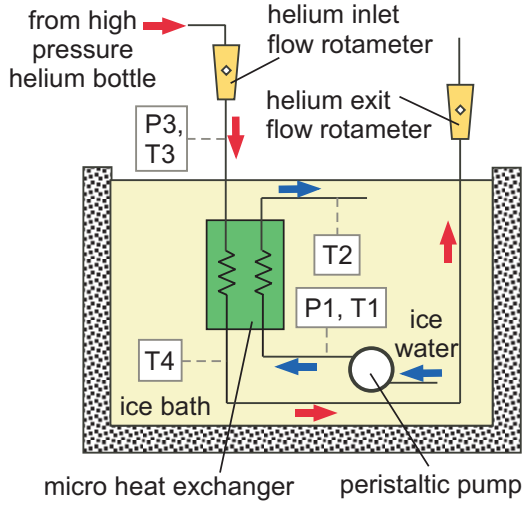

Fig. 6: Ice bath test setup of the micro heat exchanger. The temperature measurements use Type E thermocouples.

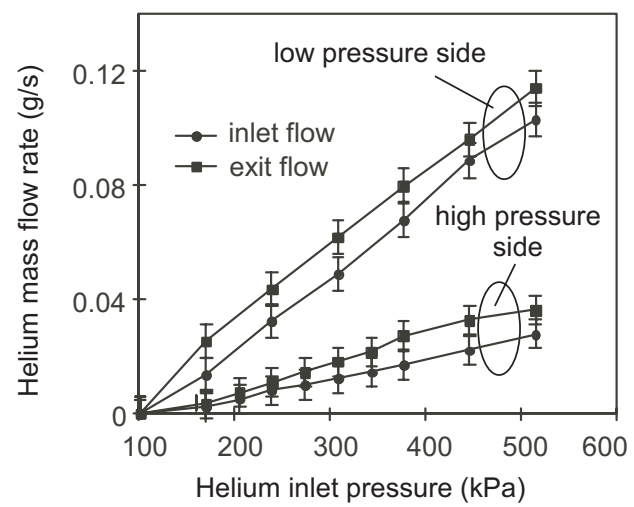

Fig. 7: Helium flow rate measured by the inlet and exit flow meters as a function of the helium inlet pressure for the high and low pressure sides.

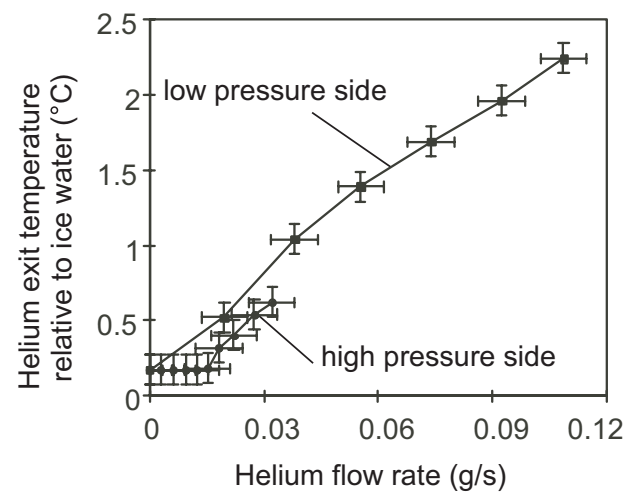

Fig. 8: Helium exit temperature, relative to measured ice water temperature, as a function of flow rate (average measured by the inlet and exit flow meters) for the high and low pressure sides. 


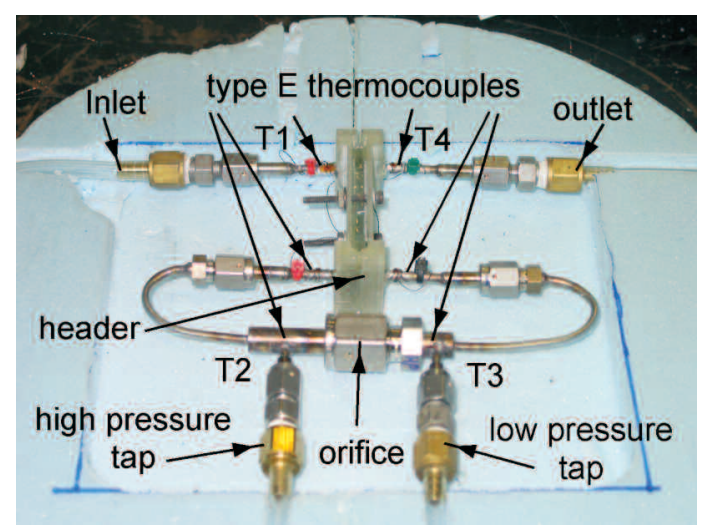

Fig. 9: Self-cooling setup. Butane gas with regulated pressure is introduced from the inlet. The micro recuperative heat exchanger is mounted inside the header. The type $\mathrm{E}$ thermocouples are inserted into the high and low pressure taps to measure the gas temperature inside the tube.

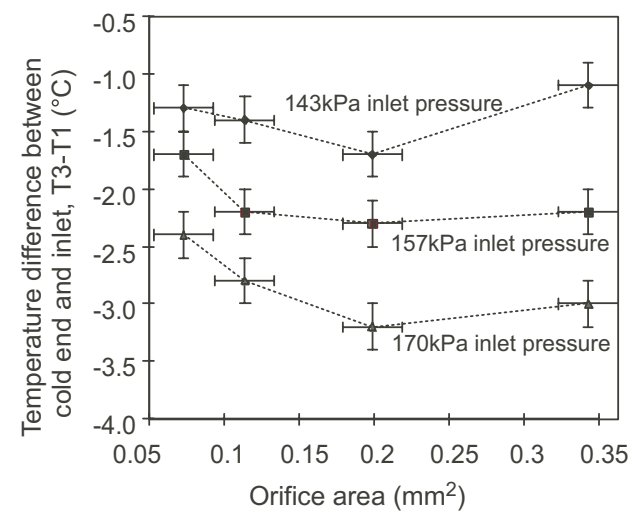

Fig. 10: Temperature difference between cold end and inlet as a function of orifice area for three different inlet pressures.

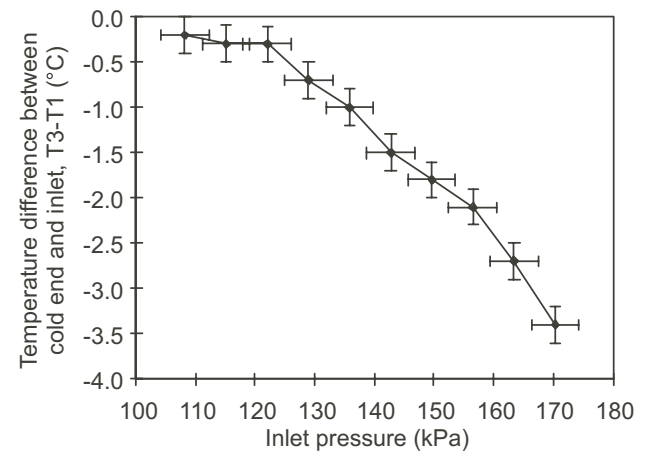

Fig. 11: Temperature difference between cold end and inlet as a function of the inlet pressure for $0.2 \mathrm{~mm}^{2}$ orifice area.

Figure 7 illustrates the inlet and outlet flow rate measured for the high and low pressure sides of the device. Note that the low pressure side of the micro heat exchanger allows much more flow for a given pressure difference due to the larger channels on the low pressure side. The inlet and exit flow rates should be the same as there was no leakage observed during the testing; however, the difference is nominally within the uncertainty of the rotameters that were used.

Figure 8 illustrates the helium exit temperature (relative to the ice water temperature) as a function of the helium flow rate (the average measured by the inlet and exit rotameters) for the two sides. The helium inlet temperature was between $17-19^{\circ} \mathrm{C}$ for all tests and therefore the helium exit temperature is an indicator of the thermal performance of the heat exchanger: a lower helium exit represents better performance. As expected, the high pressure side showed better performance than the low pressure side because the fin structure is more dense (Fig. 5). However, this difference is relatively slight because the dominant thermal resistance in the heat exchanger is the Pyrex plate.

For the second test, the device was installed into a macroscale self-cooling system (Fig. 9). In this experiment, the heat exchanger is placed in a cavity within a piece of Styrofoam insulation and surrounded by fiberglass insulation. The hot inlet is provided with a flow of butane from a high pressure bottle at room temperature. The pressure, flow rate, and temperature $\left(\mathrm{T}_{1}\right)$ of this flow are measured. The high pressure butane passes through the heat exchanger where it is pre-cooled by the low pressure butane returning from the cold end. The butane is expanded through an orifice (a precision jewel installed in a blank gasket) located at the cold end of the system; by varying the size of the orifice it is possible to control the flow rate through the heat exchanger. The temperatures on either side of the orifice $\left(\mathrm{T}_{2}\right.$ and $\left.\mathrm{T}_{3}\right)$ are measured using thermocouples that penetrate the butane stream. Figure 10 illustrates the temperature difference between the cold end and the inlet as a function of orifice area for three different inlet pressures. The optimal orifice size is $0.2 \mathrm{~mm}^{2}$. The temperature difference as a function of inlet pressure for this optimal orifice is shown in Fig. 11; the measured temperature difference matches the theoretical model assuming a parasitic heat loss of $40 \mathrm{~mW}$.

\section{DISCUSSION}

In addition to the challenges in the various steps identified in the design and fabrication section, the development of the fabrication process has yielded a number of important lessons. At a very basic level, the fragility of the $100 \mu \mathrm{m}$ thick glass plate requires it to be bonded to at least one $\mathrm{Si}$ wafer as early as possible in the process. Another general challenge is the footprint of the device, which is so large that the possibility of a defect or nonuniformity is high. In the processing steps, as already discussed, the primary challenges include the double-sided bonding of the glass wafer; the DRIE sequence and its limitations related to overall uniformity, micro loading, and footing; the frit glass bonding; and the electrochemical etching. However, reasonable solutions exist for each of these challenges, and a feasible process for manufacturing heat exchangers in this manner has been developed.

The self-cooling data is limited to very small temperature differences relative to a practical device that is useful for cryosurgery. This is due to the following reasons:

1) The difference between the high and low pressures in the heat exchanger is limited by the structural integrity of the base plate. The pressure difference anticipated for a cryosurgical probe may be as high as $1400 \mathrm{kPa}$ (200 psi) whereas the testing was limited to $70 \mathrm{kPa}(10 \mathrm{psi})$ in order to avoid fracturing the base plate. Theoretically, this thickness of the base plate should be able to sustain larger pressure differences, but in practice this is not true A thicker base plate would provide greater structural integrity, but the increased transverse thermal resistance would further reduce cooling power. This design concept is not ideal if the base plate is made of Pyrex and an alternative material with a higher conductivity (optimal value is about $30 \mathrm{~W} / \mathrm{m}-\mathrm{K}$ ) must be found to make this concept practical.

2) The fin height of $200 \mu \mathrm{m}$ is much smaller than the initial target of $500 \mu \mathrm{m}$. This modification was required by manufacturing constraints, but results in both a $2.5 \times$ reduction in heat transfer area as well as a $5 \times$ increase in the pressure drop. 
3) The structural integrity and hermeticity restrictions prevent installation in a thermal vacuum facility. Therefore, there is a substantial parasitic heat load on the device that prevents it from achieving very low temperature.

The predicted and measured effectiveness as a function of mass flow rate (based on the hot side energy balance) is illustrated in Fig. 12. The effectiveness of the heat exchanger $(\varepsilon)$ is defined as the ratio of the heat transferred within the heat exchanger to the maximum possible amount of heat that could have been transferred had the heat exchanger been perfect (i.e., the heat transferred if $\mathrm{T}_{4}$ was equal to $\mathrm{T}_{1}$ ). The effectiveness can be computed based on either the hot- or cold-side balance ( $\varepsilon_{h}$ or $\varepsilon_{c}$, respectively):

$$
\varepsilon_{h}=\frac{\dot{q}_{H X, h}}{\dot{q}_{H X, \max }} \text { or } \varepsilon_{c}=\frac{\dot{q}_{H X, c}}{\dot{q}_{H X, \max }},
$$

where $\dot{q}_{H X}$ is the actual heat transfer and $\dot{q}_{H X, \max }$ is the ideal heat transfer in perfect conditions. The error bars are theoretical errors based on the uncertainty in the thermocouple temperature measurements. Note that the error increases as the mass flow rate drops due to the smaller temperature differences that are required to compute the effectiveness. This measured result properly matches the theoretical model.

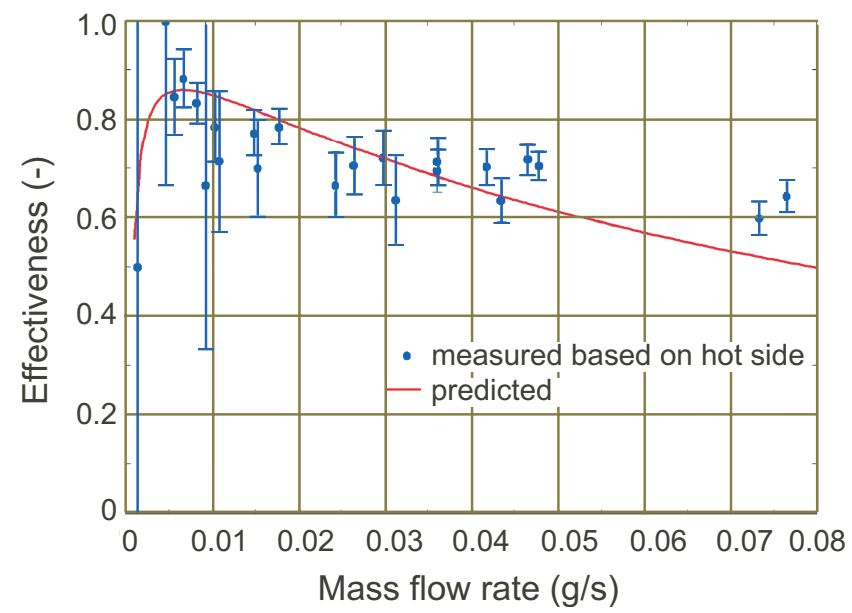

Fig. 12: Measured effectiveness based on hot side energy balance and the predicted effectiveness using the micro heat exchanger model as a function of the mass flow rate.

\section{SUMMARY}

A planar micro heat exchanger with a footprint of $6 \times 1.5 \mathrm{~cm}^{2}$ and $2.5 \mathrm{~mm}$ thickness was fabricated in a 5 -mask process using 3 glass wafers and $2 \mathrm{Si}$ wafers. In our tests, the self-cooling performance of the micro heat exchanger was mainly limited by (i) mechanical robustness of the glass base plate and (ii) Si fin height that was constrained by the uniformity of DRIE. However, the effectiveness of the heat exchanger varied from 0.8 at a mass flow rate of about $0.01 \mathrm{~g} / \mathrm{s}$ to 0.6 at $0.075 \mathrm{~g} / \mathrm{s}$, which matched the developed theoretical model. We are presently developing a new structure to avoid fracturing the base plate. If successful, the robustness, flexibility and performance associated with the micromachined device may result in the application of cryosurgery to new biomedical areas.

\section{ACKNOWLEDGEMENT}

This work was funded in part by a grant from the US National Institutes of Health (R21 EB003349-02).

\section{REFERENCES}

[1] J. Dobak, "A Review of Cryobiology and Cryosurgery," Advances in Cryogenic Engineering, 43, pp. 889-896, 1998

[2] Z. H. Chang, J. J. Finkelstein, J. G. Baust, "Optimization of Cryosurgical Instrumentation for use in Minimally Invasive Prostate Surgery," ASME Heat Transfer Div Publ HTD, v 267, pp. 45-55, 1993

[3] B. Z. Maytal, "Fast Joule-Thomson Cryocycling Device for cryosurgical Applications," Advances in Cryogenic Engineering, 43, pp. 911-917, 1998

[4] J. Dobak, X. Yu, and K. Ghaerzadeh, "A Novel Closed Loop Cryosurgical Device," Advances in Cryogenic Engineering, 43, pp. 897-902, 1998

[5] R. Masumoto, K. Oshio, F. A. Jolesz, "Monitoring of Laser and Freezing-Induced of the Liver with Tl-Weighted MR Imaging," Journal of Magnetic Resonance Imaging, 2, pp. 555, 1992

[6] B. Rubinsky, J. C. Gilbert, G.M. Onik, M.S. Roos, S.T. Wong, K.M. Brennan, "Monitoring Cryosurgery in the Brian and Prostate with Proton NMR," Cryobiology, 30, pp. 191-199, 1993.

[7] G.R. Pease, S.T. Wong, M.S. Roos, B. Rubinsky, "MR ImageGuided Control of Cryosurgery," Journal of Magnetic Resonance Imaging, 5, pp. 753-760, 1995

[8] J. K. Cohen, R. J. Miller, "Thermal Protection of Urethra during Cryosurgery of the Prostate," Cryobiology, 31, pp. 313-316, 1994

[9] A. Gage, "Five-year Survival Following Cryosurgery for Oral Cancer," Archives of Surgery, 111, pp. 990-994, 1976

[10] A.A. Gage, J.A. Caruana and M. Montes, "Critical Temperature for Skin Necrosis in Experimental Cryosurgery," Cryobiology, 19, pp. 273-282, 1982

[11] J. Bischoff, K. Christov, B.A. Rubinsky, "Morphological Study of Cooling Response in Normal and Neoplastic Human Liver Tissue: Cryosurgical Implications," Cryobiology, 30, pp. 482-492, 1993

[12] A. A. Gage, K. Guest, M. Montes, J.A. Caruana, D. A. Whalen, Jr., "Effect of Varying Freezing and Thawing Rates in Experimental Cryosurgery," Cryobiology, 22, pp. 175-182, 1985

[13] A. A. Gage, "Cryosurgery in the Treatment of Cancer," Surgery, Gynecology \& Obstetrics, 174, pp. 73-92, 1992

[14] E. D. Marquardt, R. Radebaugh, J. Dobak, "A cryogenic Catheter for Treating Heart Arrhythmia," Advances in Cryogenic Engineering, 43, pp. 903-910, 1998.

[15] G. F. Nellis, "A Heat Exchanger Model that Includes Axial Conduction, Parasitic Heat Load, and Property Variations," Cryogenics, vol. 43, no. 9, pp. 523-538, 2003

[16] M. Frank, M, Recuperative Heat Exchanger for a MEMS Cryoprobe, M.S. Thesis, Univ. Wisconsin, Dept. of Mechanical Engineering, 2004

[17] K. Ishihara, C. F. Tung, A. A. Ayón, "An Intertial Sensor Technology Using DRIE and Wafer Bonding with Interconnecting Capability," J. Micromech. Sys., 8 (4), pp. 403-408, 1999

[18] K. Kühl, S. Vogel, U. Schaber, R. Schafflik, B. Hillerich, "Advanced Silicon Trench Etching in MEMS Applications," SPIE, vol. 3511, Santa Clara, California, pp. 97-105, 1998

[19] M. Chabloz, J. Jiao, Y. Yoshida, T. Matsuura, K. Tsutsumi, "A Method to Evade Microloading Effect in Deep Reactive Ion Etching for Anodically Bonded Glass-silicon Structures," IEEE Intl. Conf. on Micro Electro Mechanical Systems (MEMS'00), Miyazaki, Japan, pp. 283-287, 2000

[20] V. Fascio, R. Wüthrich, D. Viquerat, H. Langen, "3D Microstructuring of Glass Using Electrochemical Discharge Machining (ECDM)," International Symposium on Micromechatronics and Human Science, pp. 179-183, 1999 